\title{
New Empirical Fitting Functions of the Lick/IDS indices using MILES
}

\author{
J. M. Martín-Hernández ${ }^{1}$, E. Mármol-Queraltó ${ }^{1}$, J. Gorgas ${ }^{1}$, \\ N. Cardiel $^{1}$, P. Sánchez-Blázquez ${ }^{2}$, A. J. Cenarro ${ }^{3}$, R. F. Peletier ${ }^{4}$, \\ A. Vazdekis ${ }^{3}$ and J. Falcón-Barroso ${ }^{5}$ \\ ${ }^{1}$ Dpto. Astrofísica, Universidad Complutense de Madrid, Avda. Complutense s/n, \\ 28040 Madrid, Spain \\ ${ }^{2}$ Centre for Astrophysics, University of Central Lancashire, Preston PR1 2HE, \\ United Kingdom \\ ${ }^{3}$ Instituto de Astrofísica de Canarias, Vía Láctea s/n, 38200, La Laguna, Spain \\ ${ }^{4}$ Kapteyn Astronomical Institute, University of Groningen, 9700 AV Groningen, \\ The Netherlands \\ ${ }^{5}$ Sterrewacht Leiden, Niels Bohrweg 2, 2333 CA, Leiden, The Netherlands
}

\begin{abstract}
Here we present new empirical fitting functions for the Lick/IDS line-strength indices as measured in MILES (Medium-resolution INT Library of Empirical Spectra; Sánchez-Blázquez et al., Cenarro et al. 2007). Following previous work in the field (Gorgas et al. 1993; Worthey et al. 1994; Worthey \& Ottaviani 1997), these functions describe the empirical behavior of the line-strength indices with the atmospheric stellar parameters. In order to derive the fitting functions we have devised a new procedure which, being fully automatic, provides a better description of the line-strength index variations in the stellar parameter space.
\end{abstract}

Keywords. stars: abundances, fundamental parameters - galaxies: stellar content - globular clusters: general

\section{The new method}

The moving-boxes method is an automatic procedure which avoids the subjectivity in the choice of stellar parameters intervals to derive fitting functions. The procedure consists in the fitting of local polynomials (up to the 2nd degree in the three atmospheric stellar parameters $)$ in a narrow temperature window $(\Delta \theta=0.2)$. These windows are moved at small steps $(\Delta \theta=0.001)$ covering the whole temperature interval. When necessary the fits are computed independently for two gravity intervals (dwarfs and giants). Finally, the predicted index for a given set of stellar parameters is derived from a weighted average of the fitting functions corresponding to all the moving boxes in which the input parameters were included.

\section{An example: empirical fitting functions for $\mathbf{H} \delta_{\mathrm{A}}$ index}

As an example of this work, we present the comparison between the computed fitting functions by Worthey et al. (1994) using Lick's library (Figure 1, left pannels) and using the new method over the MILES library (Figure 1, right pannels). 

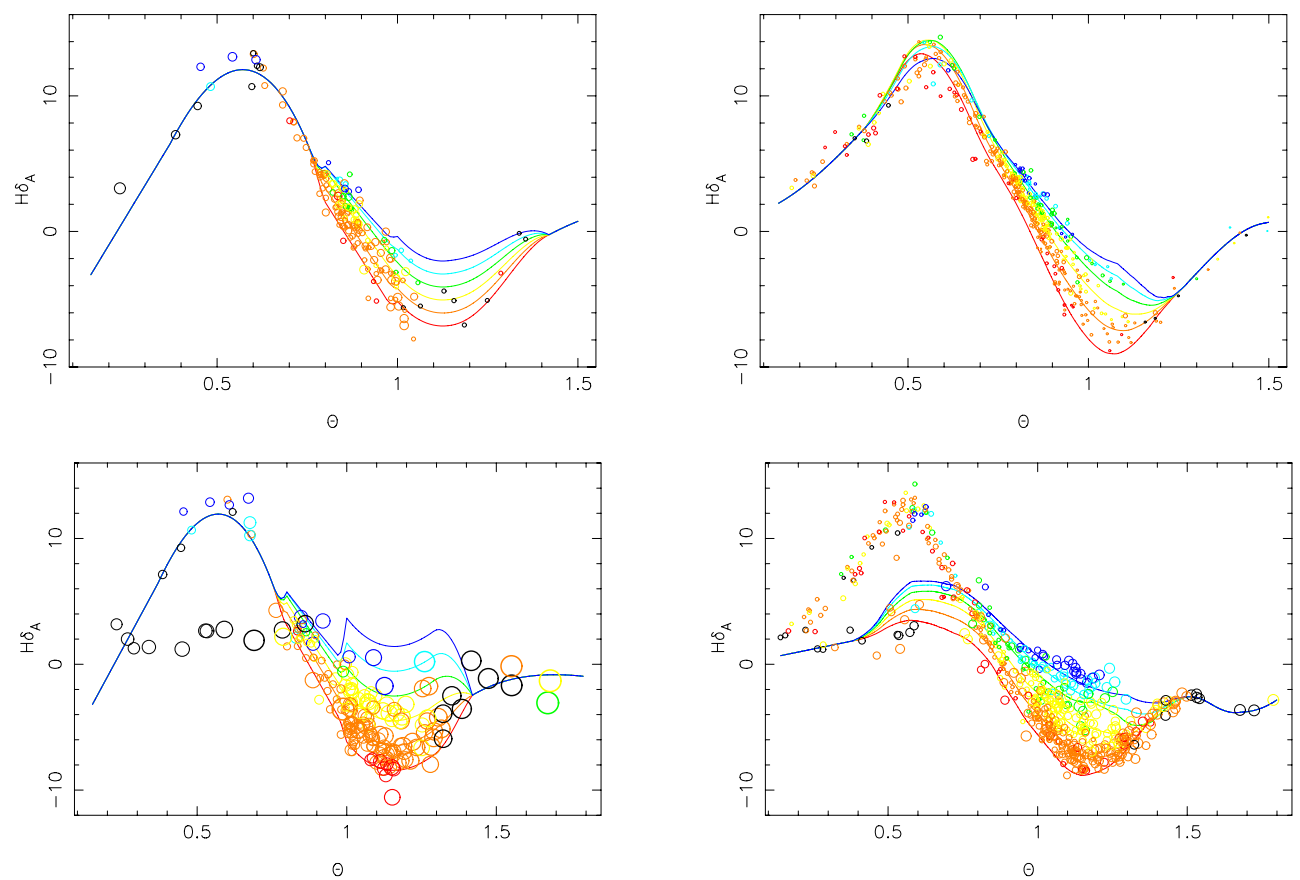

Figure 1. Left: Empirical calibration of $\mathrm{H} \delta_{\mathrm{A}}$ index presented by Worthey \& Ottaviani (1997) using the original Lick/IDS library data. Right: Empirical calibration of $\mathrm{H} \delta_{\mathrm{A}}$ index computed with the new method based on moving boxes using the MILES library. In both cases we present separately the empirical fitting functions for dwarf (upper pannels, fitting lines for $\log g=4.5$ dex) and giant (lower pannels, fitting lines for $\log g=2.0 \mathrm{dex}$ ) stars.

\section{Future work}

These empirical fitting functions have been computed at the spectral resolution of MILES $(2.3 \AA)$. We are now in the process of introducing the spectral resolution as a fourth parameter. The future release of the fitting functions will allow a direct comparison of the model predictions with spectra at any given instrumental resolution and intrinsic velocity dispersion (above the nominal resolution of MILES).

\section{References}

Gorgas, J., Faber, S.M., Burstein, D., Gonzalez, J.J., Courteau, S., \& Prosser, C. 1993, ApJS 86,153

Sánchez-Blázquez, P., Peletier, R.F., Jiménez-Vicente, J., Cardiel, N., Cenarro, A.J., FalcónBarroso, J., Gorgas, J., Selam, S.O., \& Vazdekis, A. 2006, MNRAS 371, 703

Cenarro, A.J., Peletier, R.F., Sánchez-Blázquez, P., Selam, S.O., Toloba, E., Cardiel, N., Falcón-

Barroso, J., Gorgas, J., Jiménez-Vicente, J., \& Vazdekis, A. 2007, MNRAS 374, 664

Worthey, G., Faber, S.M., Gonzalez, J.J., \& Burstein, D. 1994, ApJS 94, 687

Worthey, G. \& Ottaviani, D. L. 1997, ApJS 111, 377 